\title{
Improved accuracy of myocardial blood flow quantification by first pass perfusion MR when corrected for steady state $\mathrm{T} 1$ relaxation
}

\author{
Niloufar Zarinabad ${ }^{1 *}$, Eva Sammut ${ }^{1}$, Tobias Voigt ${ }^{4}$, Gilion Hautvast ${ }^{5}$, Marcel Breeuwer ${ }^{2,3}$, Reza Razavi ${ }^{1}$, Eike Nagel ${ }^{1}$, \\ Valentina O Puntmann ${ }^{1}$, Amedeo Chiribiri ${ }^{1}$
}

From 16th Annual SCMR Scientific Sessions

San Francisco, CA, USA. 31 January - 3 February 2013

\section{Background}

Quantification of myocardial blood flow (MBF) on firstpass dynamic contrast enhanced (DCE) MR perfusion imaging has been performed by several different methods. However MBF estimates are dependent on the analysis model used, as the absolute concentration of the contrast agent during first pass is not known. To improve the accuracy of the MBF estimates calibration is required. We propose a novel approach for the quantification of MBF based on patient-specific calibration using steady state $\mathrm{T} 1$ relaxation values (1) (figure 1).

\section{Methods}

T1 weighted images were acquired before and after contrast injection to perform steady state measurements of myocardial blood volume (MBVss) (2): MBVSS = (1/T1post contrast $-1 / \mathrm{T} 1$ pre contrast) tissue / (1/T1post contrast $-1 /$ T1 pre contrast) blood.

Based on the first pass perfusion data, MBVDCE and MBFDCE were obtained using Fermi deconvolution. The calibration factor $(\mathrm{CF})$ was calculated as $\mathrm{CF}=$ MBVss / MBVDCE and used to calibrate MBF values (MBFcalib) according to: $\mathrm{MBF}$ calib $=$ MBFDCE *CF.

Perfusion data were obtained from five patients using a dual-bolus injection of $0.075 \mathrm{mmol} / \mathrm{kg}$ Gadobutrol (Gadovist, Bayer, Germany) injected at $4 \mathrm{ml} /$ minute followed by a $20 \mathrm{ml}$ saline flush using a Philips Achieva 3T (TX) system, equipped with a 32-channel cardiac phased array receiver coil (Philips, Best, Netherlands). Hyperaemia was induced with adenosine administered at 140 $\mathrm{mcg} / \mathrm{kg} / \mathrm{min}$. First-pass perfusion images were acquired

${ }^{1}$ Imaging Sciences and Biomedical Engineering, Kings College London, London, UK

Full list of author information is available at the end of the article using a saturation recovery gradient echo method (TR/ TE $3.0 \mathrm{~ms} / 1.0 \mathrm{~ms}$, flip-angle $15^{\circ}$; effective k-t SENSE acceleration 3.8 , spatial resolution $1.2 \times 1.2 \times 10 \mathrm{~mm}$, saturationrecovery delay $120 \mathrm{~ms}$ ). T1 images were acquired using a cardiac triggered Modified Look Locker Inversion Recovery (MOLLI) sequence(slice thickness $8 \mathrm{~mm}$, FOV $388 \times 320 \mathrm{~mm} 2$, matrix $216 \times 216,11$ different TI, range 90ms-3s). T1 was estimated through fitting of a three

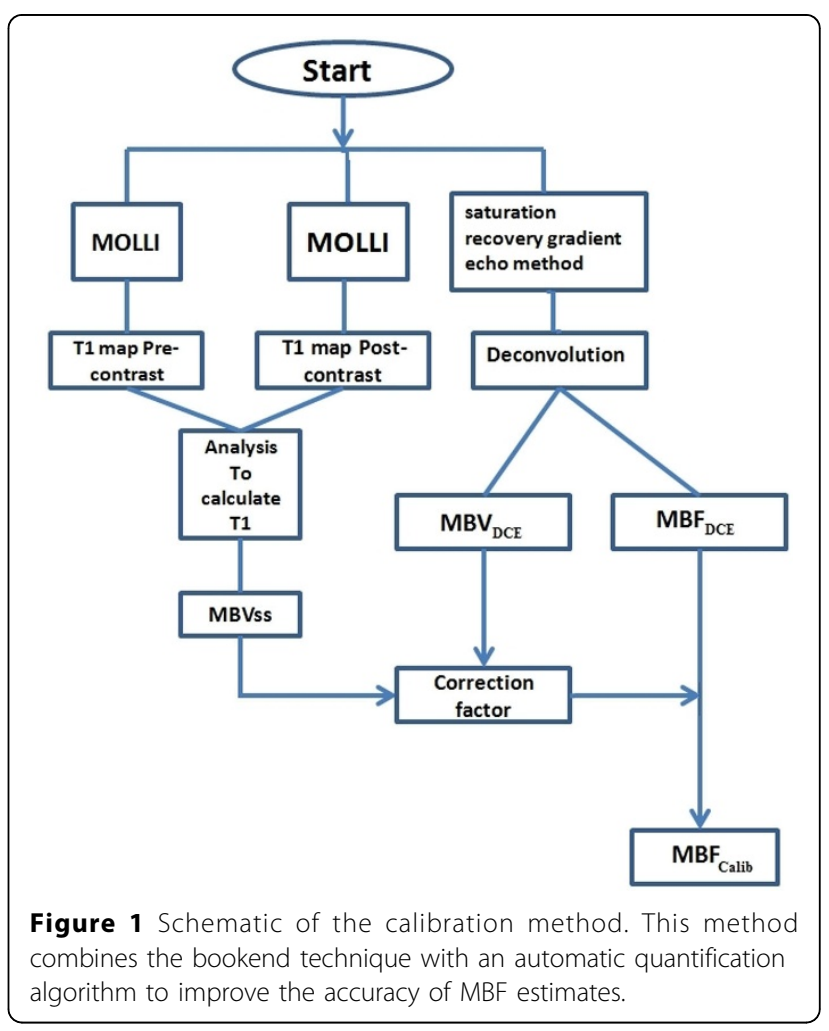



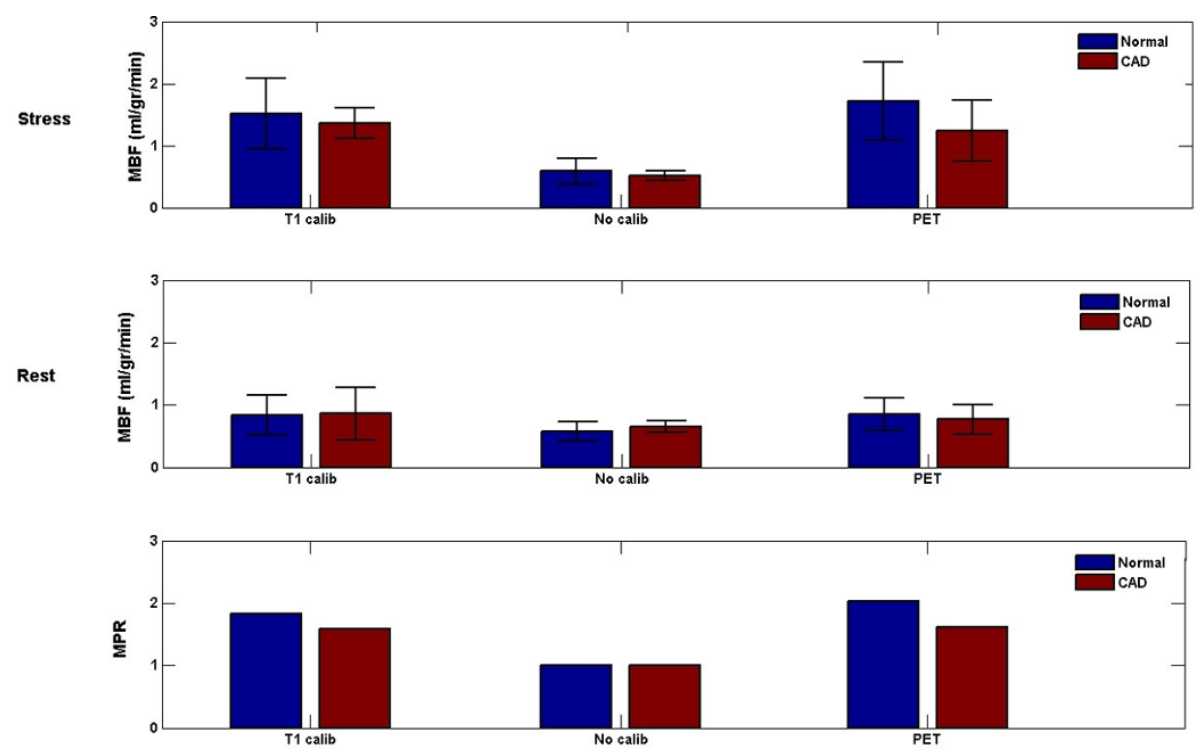

Figure 2 Comparison between MBFcalib ( $\mathrm{ml} / \mathrm{gr} / \mathrm{min}$ ) values determined by the novel T1 calibration algorithm (T1 calib), the MBF values obtained from standard non calibrated algorithm (no calib) and reference values from literature in normal (Blue) and ischemic (red) myocardial regions. The calibrated measurements agree with literature values.

parameter exponential model $\mathrm{SI}(\mathrm{t})=\mathrm{A}-\mathrm{B} \exp \left(\mathrm{t} / \mathrm{T} 1^{*}\right)$ and Look-Locker correction $\mathrm{T} 1=\mathrm{T} 1 \%((\mathrm{~B} / \mathrm{A})-1)$.

\section{Results}

The bar plot in figure 2 compares the rest and stress mean MBFcalib values with the mean MBFDCE values and the reference perfusion values reported in the literature (3) in normal and ischaemic myocardial regions. The mean stress values of MBFcalib for both normal and abnormal regions $(1.35 \pm 0.24 \mathrm{ml} / \mathrm{gr} / \mathrm{min}$ for ischemic and $1.51 \pm 0.56 \mathrm{ml} / \mathrm{gr} / \mathrm{min}$ for normal region) are in closer agreement with stress PET values $(1.24 \pm 0.49 \mathrm{ml} / \mathrm{gr} / \mathrm{min}$ for ischemic and $1.73 \pm 0.63$ ) compared with the MBFDCE values obtained from non calibrated data $(0.58 \pm 0.207 \mathrm{ml} /$ $\mathrm{gr} / \mathrm{min}$ for ischemic and $0.65 \pm 0.070$ for normal).

\section{Conclusions}

The measurements of quantitative MBF agree closely with previously published gold-standard measurements by PET after calibration is performed. The addition of T1 steady state measurements to DCE measurements of MBF improve the accuracy of the measurement as inter-subject variability is taken into account.

\section{Funding}

The authors acknowledge financial support from the Department of Health via the National Institute for Health Research (NIHR) comprehensive Biomedical Research Centre award to Guys and St Thomas NHS
Foundation Trust in partnership with Kings College London.

\section{Author details}

${ }^{1}$ Imaging Sciences and Biomedical Engineering, Kings College London, London, UK. ${ }^{2}$ Imaging Systems, MR, Philips Healthcare, Best, the Netherlands. ${ }^{3}$ Biomedical Engineering,Biomedical Image Analysis, Eindhoven University of Technology, Eindhoven, the Netherlands. ${ }^{4}$ Clinical Research Europe, Philips Research, Aachen, Germany. ${ }^{5}$ Healthcare Incubators, Philips Innovation Group, Eindhoven, the Netherlands.

Published: 30 January 2013

\section{References}

1. Shin W:. Magn Reson Med 2007.

2. Bauer W:. Magn Reson Med 1996.

3. Morton G.. JACC 2012.

doi:10.1186/1532-429X-15-S1-P85

Cite this article as: Zarinabad et al:: Improved accuracy of myocardial blood flow quantification by first pass perfusion MR when corrected for steady state T1 relaxation. Journal of Cardiovascular Magnetic Resonance 2013 15(Suppl 1):P85. 